\title{
Interdisciplinarity and Integration: An Introduction to the Special Issue on Psychopathology in Medical Settings
}

\author{
Golan Shahar ${ }^{1}$ (1) \\ Accepted: 7 November 2020 / Published online: 20 November 2020 \\ (c) Springer Science+Business Media, LLC, part of Springer Nature 2020
}

\begin{abstract}
As the world views, incredulously, the calamitous consequences of the COVID-19 pandemic, the inseparable connections between body and mind become more and more apparent, even for the heretics (i.e., biological determinists). Such realizations also bolster the understanding of the close link between medical conditions and psychopathology. Launched prior to the COVID-19 epidemic, this special issue sets out to illuminate the prevalence, course, etiology, and responses to a myriad of psychopathological conditions in medical conditions. The 13 articles in this special issue address a variety of medical conditions (chronic illness and chronic pain, Pica, cancer, acute delirium, factitious disorders, functional neurological symptoms, sleep disorders, fetal conditions), mental disorders (depression, anxiety, suicidality, eating disorders, personality disorders, PTSD), medical settings (primary care vs. specialty clinics), and developmental levels (children, adolescents, and adults). The overarching theme emanating from reading these articles is that clinical-health psychology, or clinical psychology in medical settings, is an ever-needed field of inquiry, epitomizing interdisciplinarity and science/practice integration.
\end{abstract}

Keywords Psychopathology $\cdot$ Medical settings $\cdot$ Development $\cdot$ Interventions $\cdot$ Integration

The entire world views, quite incredulously, the calamitous consequences of the COVID-19 pandemic. As of writing these words (October the 17th, 2020), about 39,666,085 individuals have been infected with coronavirus worldwide, and 1,116,576 died from it. Millions lost their jobs due to economies closing in the face of lockdowns, others, seemingly recuperating, are discovering the challenge of Long Covid (long term effect of the coronavirus disease; https ://www.gov.uk/government/publications/covid-19-longterm-health-effects/covid-19-long-term-health-effects). It is therefore not surprising-albeit most pertinent to the aims of this journal-that a mental disorder pandemic is increasingly surfacing. Namely, rates of depression, anxiety, suicidality, and externalizing psychopathology (e.g., domestic violence) are on the rise in various places around

The author wishes to thank Dr. Ronald Brown, Editor in Chief of JCPMS, for his encouragement and support during guest-editing this special issue.

Golan Shahar

Golab.shahar878@gmail.com; shaharg@bgu.ac.il

1 Department of Psychology, Ben-Gurion University of the Negev, Beersheba, Israel the world (Bareket-Bojmel, Shahar, \& Margalit, 2020, https ://www.who.int/teams/mental-health-and-substance-use/ covid-19). For those of us working in the field of clinical psychology in medical settings, this is hardly surprising, as we have long internalized the inseparability of body and mind, as well as the close link between medical conditions and psychopathology.

The purpose of this special issue, launched prior to the onset of COVID-19, is to illuminate the prevalence, course, etiology, and responses to a myriad of psychopathological conditions in medical conditions. That this prevalence is quite high is quite well known. Focusing on unipolar depression as an illustration, it has been shown that up to $23 \%$ of individuals inflicted by chronic physical illness also experience clinically significant depression (Katon, 2011; Shahar, Lassri, \& Luyten, 2014). Similar high prevalences have been reported for anxiety and suicidality. Nevertheless, other disorders, less common than depression and anxiety, pose a challenge to psychologists working in medical settings, e.g., PTSD, eating disorders, suicidality, etc. Conversely, individuals with mental disorders may pose serious challenges to physicians aiming at treating their physical ailments (Barber $\&$ Thornicroft, 2018). We were therefore not surprised to receive a large number of submissions in response to our 
call for papers on psychopathology in medical settings andthrough a stringent review process-were pleased with the 13 articles meeting criteria for publication.

In the first article, Karoly (2020) applies his evolving, Goal-Centered, Self-Regulatory, Automated, Social Systems Psychology (GRASSP) model (e.g., Karoly, 2018), to understand psychopathology-primarily depression and anxiety - that result from chronic physical pain. GRASSP includes cognitive, motivational (goal-oriented), and selfregulatory components that aim at shedding light on how people adopt - or not- to the complex, undeniably somatic, experience of chronic pain, where "adaptation" is primarily defined by Karoly as the ability to pursue relevant goals. To the extent that adaptation is not attained, depression and anxiety ensue. I find the focus on motivation refreshing in a field that construe chronic pain in almost exclusively biological and cognitive terms. Furthermore, Karoly's article illustrates the feasibility — and importance-of linking profound theory, a review of empirical findings, and clinical wisdom into a coherent framework.

Rodrigues, Shih, and Cohen (2019) apply a biopsychosocial model of PICA in children with Sickle Cell Disease. Pica is the developmentally inappropriate consumption of non-nutritive items for at least 1 month (American Psychiatric Association, 2013). Sickle cell disease is a genetic disorder characterized by anemia, decreased oxygen delivery, and tissue hypoxemia due to vaso-occlusion. Rodrigues et al. (2019) cite studies attesting to a high prevalence of Pica in pediatric sickle cell disease and review research on the biological and psychosocial correlates of Pica. Interestingly, Pica sufferers who have sickle cell disease exhibit a heterogenic combination of these correlates. Directions for intervention along behavioral, biological, sensory, and environmental routes are proposed.

The third article in this special issue focuses on neurologic cancer and espouses an intriguing existential perspective. Loughan et al. (2020) conduct a systematic literature review of articles focusing on fear of progression, recurrence, and death in patients diagnosed with primary brain tumor (BPT). After narrowing-down the number of publications (from 1555 to 8!) using stringent criteria, Loughan et al. (2020) identify important themes that underlie these articles. In reading through the authors' finding, it was impressive to learn that fear of death, experienced in a onethird PBT patients, is strongly associated with depression severity. To date, I am not aware of extant, evidence-based protocols for chronic, terminal or non-terminal, medical illness, that factor-in death anxiety in the treatment of depression, despite clear recommendations by proponents of the existential perspective (Yalom, 1980). This is certainly an oversight that needs to be rectified in the future.

Bolton, Thilges, Lane, Lowe, and Mumby (2020) examine PTSD in hospitalized patients experiencing acute delirium.
Delirium pertains to disturbance in attention, awareness, and other cognitive factors, with a rapid onset and fluctuating course. According to Bolton et al. (2020), the prevalence of delirium ranges between 14 and $24 \%$. There is almost no systematic research on PTSD following delirium, but Bolton et al. (2020) cite a series of case studies attesting to the feasibility of this phenomenon (i.e., PTSD following delirium). Bolton et al. (2020) further distinguish the occurrence of this phenomenon in inpatient unites vs. intensive care units, and younger and older adults, and also discuss possible risk and resilience factors, and point to some innovative directions for intervention.

Sim, Weber, Harrison, and Peterson (2020) examine a very intriguing linkage, i.e., between chronic pain and eating disorders. Despite a relative dearth of research evidence on eating disorders in chronic pain, the effect of such pain on eating disruption is well known. These disruptions include, but are not limited to, appetite change, dietary restrictions, nutrition deficiencies, and reduced activities related to eating. These behaviors often lead to weight loss. According to Sim et al. (2020), dietary restrictions in chronic pain may look similar to that occurring in Anorexia Nervosa (AN), and is described under the label Avoidant Restrictive Food Intake Disorder (ARFID). Sim et al., review temperamental, behavioral, and neurobiological risk factors linking chronic pain and eating disorders, and propose central sensitization as an underlying mechanism. Central sensitization pertains to the central nervous system being provoked into a persistent state of heightened reactivity rendering individuals highly sensitive to emotional and somatic input. It is recognized to account for the development and maintenance of chronic pain (Latremoliere \& Woolf, 2009; Woolf, 2011). Sim et al. (2020) propose that to manage high levels of sensitivity, individuals may develop unique patterns of behavioral avoidance, pain behaviors, and/or restrictive eating. Impressively, Sim et al. (2020) conclude with suggestions for transdiagnostic assessment and intervention strategies to address sensitivity in eating disorders and chronic pain.

Moving from specialty clinics and ICUs, Costello et al. (2020) address the gnawingly important issue of adolescent depression in primary care. Unipolar depression is highly prevalent in adolescence and often poses serious challenges to pediatricians and adolescent medicine physicians. Arguably, this is why the American Academy of Pediatrics' (AAP) Guidelines for Adolescent Depression in Primary Care (GLAD-PC) were created to enhance the early identification and treatment of adolescent depression (Zuckerbrot, Cheung, Jensen, Stein, \& Laraque, 2007). Costello et al. (2020) described an initiative launched by integrated psychologists in a pediatric primary care setting, where they partnered with pertinent providers and clinical staff to implement a screening/early identification and management procedure. Avoiding spoilers, I strongly encourage the readers 
to examine carefully the procedure described in this article, which strongly attests to the centrality and effectiveness of integrated psychologists in pediatric primary care settings, particularly with respect to the identification and management of depression.

Bursch, Emerson, and Sanders (2020) target an enormously challenging problem to medical providers: Factitious Disorder Imposed on Another (FDIA). The latter title refers to the psychopathology of the perpetrator of Munchausen By Proxy (MBP), pertaining to individuals who abusively and compulsively falsify physical, psychiatric, or developmental disorders in a child or adult victim in order to satisfy a psychological need. Bursch et al. (2020), leading experts in the field, review the extant literature on the manifestation and epidemiology of both FDIA and MBP, and then build on this literature by focusing on the role of psychologists in medical settings with respect to FDIA/MBP. Such roles include the following tasks: identifying victims and/or abusers, assessing occurrence and damage, evaluating and intervening in the impact of victim-abuser separation, and managing the entire case. Guidelines for each of these tasks are provided. I expect that psychologists in medical settings who-intentionally or not-confront FDIA/MBP will find this article invaluable.

Returning to chronic pain, Weisfeld and Dunleavy (2020) offer a unique combination of a review/theory and a rare longitudinal case study. The focus of this article is on the link between chronic pain and post-traumatic stress disorder (PTSD). There is a very high comorbidity between the two conditions, with a bidirectional influence (Otis, Keane, Kerns, Monson, \& Scioli, 2009). Weisfeld and Dunleavy (2020) describe four theoretical models of the link between chronic pain and PTSD: Mutual Maintenance (i.e., several factors contribute to the chronic nature of both), Shared Vulnerability (anxiety sensitivity as a risk factor for the onset of both conditions), Fear Avoidance (a maladaptive coping strategy leading to both conditions), and Triple Vulnerability (interactions between biological vulnerability, general psychological vulnerability, and a specific, within-individual vulnerability propelling the sufferer to focus on situational reminders). Presenting research findings supportive of all four models, Weisfeld and Dunleavy (2020) describe a unique case study of an adult patient suffering from musculoskeletal pain and delayed-onset PTSD. Extensive medical records from a very wide array of therapies provided to the patient over 10 year were brought to bear on the four models, providing primary support for the Mutual Maintenance Model. The authors demonstrate compellingly that, although a case study cannot confirm a theory/model, it may serve as a powerful vehicle for stirring research attention to a certain theory or model.

Weiss et al. (2020) focus on pediatric functional neurological symptom disorder (FNSD, previously known as conversion disorder). FNSD pertains to children/adolescents presenting with debilitating, unexplained physical symptoms, and - as the authors suggest—are likely to confront psychologists in specialty clinics, emergency departments, and inpatient settings. The condition is highly impairing (Bolger, Collins, Michels, \& Pruitt, 2018) and costly (Doss $\&$ Plioplys, 2018). Weiss et al. (2020) review pertinent research on FNSD, present various etiological models, and provide treatment recommendations. In reading the article, I was taken by three features gleaned from the focus on FNSD: the multi-dimensionality of all etiological models presented (which include elements of brain, behavior, and interpersonal relationships), the centrality of self-related processes in the development of the condition (particularly self-focus attention, self-agency), and the diverse treatment that is needed (psychotherapy, pharmacotherapy, occupational therapy). All three aspects demand from clinical psychologists working in medical settings a considerable clinical, intellectual, and interpersonal acumen in managing FNSD.

The subsequent article is also focused on children, albeit on preschoolers. Fehr, Chambers, and Ramasani (2020) illuminate the relationships between two well-known pediatric conditions: sleep difficulties and anxiety. The close link between the two conditions is also quite well known, although — as noted by Fehr et al. (2020) — anxiety is rarely considered in the literature on young (i.e., preschool) children (see Kushnir \& Sadeh, 2011). Espousing a pragmatic focus, Fehr et al. (2020) review the literature on the anxiety-sleep difficulties link that leads directly to recommendations for evidence-based interventions in young children. I was particularly struck by the centrality of the interpersonal context of both conditions (e.g., separation anxiety is a risk factor for sleep difficulties), as well as by the bidirectional nature of the anxiety-sleep link (i.e., sleep disorders in young children predict anxiety disorders later on in life). Similarly, the centrality of behavioral interventions delivered to the child through the caregiver is also noteworthy in Fehr et al. (2020). The authors also note other promising avenues for intervention (e.g., bibliotherapy) and point out useful future directions for research and practice.

Potthoff, Palma, Davidson, Bujoreanu, and Thompson (2020) target one of the most classic examples of psychopathology in medical settings, namely, eating disorders. More specifically, Potthof et al. (2020) focus on hospitalized patients with eating disorders and describe the challenges faced by clinical psychologists working with these patients and their families. Of the various challenges described, I am particularly taking note of interdisciplinarity: The various eating disorders are, by definition, complex, biopsychosocial entities that necessitate the synergistic involvement of psychologists, psychiatrists, primary care physicians, nutritionists, occupational therapists, etc. It is very difficult for 
psychologists to find their place within such a diverse team, but - as the cases presented in this article attests, this is both possible and critical for the well-being of eating disorder patients and their families.

Dempsey, Chavis, Willis, Zuk, and Cole (2020) focus on the mental health needs of expectant parents who deal with fetal anomaly during pregnancy. These parents are documented to suffer from a host of psychiatric symptoms (i.e., depression, anxiety, traumatic stress) and to experience disruptions in spousal/family life. Although psychologists have not yet been listed as essential team members in fetal care centers (Moon-Grady et al., 2017), Dempsey et al. (2020) argue-quite cogently - that psychologists are uniquely qualified to contribute to parents' mental health due to their skills in screening and assessment, clinical intervention, and consultation-liaison. An additional contribution of psychologists to fetal care center is educating staff in these centers about biopsychosocial processes that affect parents, as well as supporting staff members who are affected by vicarious trauma emanating from working with high-risk parents who go through traumatic birth or fetal/neonatal loss (Beck \& Gable, 2012). As is the case with the other articles, interdisciplinarity, broad knowledge in psychopathology and behavioral medicine, and a flexible, integrative, evidencebased is required from psychologists in fetal care centers.

The final article in this special issue is by Rogers, Joiner, and Shahar (2020), who focus on suicidality among individuals with chronic physical illness. Individuals suffering from chronic physical illness (cancer, pain conditions, asthma, diabetes, etc.) are at a heightened risk for suicidal ideations, attempts, and deaths (Ahmedani et al., 2017). This is partly because of comorbid mental health conditions such as depression, anxiety, and substance abuse, although the latter do not fully account for the above-noted heightened risk for suicidality in individuals who are chronically physically ill. Rogers et al. (2020) present a unified and integrative theoretical perspective that relies on three psychological pillars: cognition, affect, and interpersonal relationships. Cognitive structures accounting for suicidality in chronic physical illness pertain to negative thoughts about self (e.g., self-criticism) and world (catastrophizing). Affect dysregulation is another serious risk factor for suicidality in this context. From an interpersonal perspective, perceived burdensomeness and thwarted belongingness, comprised of two proximal causes for death by suicide in Joiner's Interpersonal-Psychological Theory of Suicide (Joiner, 2005), are posited to be highly operative in the context of chronic physical illness. To these two interpersonal factors, the authors also add critically expressed emotion, a family-based, toxic interpersonal exchange that is known to produce and maintain psychopathology and physical illness. Rogers et al. (2020) conclude by drawing implications from this unified cognitive-affective-interpersonal perspective, with a particular attention to the unique context of chronic physical illness.

Assuming a bird's eye view of all 13 articles, several common themes surface. First, each of the articles speaks to the centrality of clinical-health psychology—or clinical psychology in medical settings - as a vanguard in both professional and scientific psychology. Clinical psychologists in medical settings shoulder a tremendously complex burden of dealing with mental health conditions embedded within a purely medical context and establishment. This challenge psychologists to acquire and polish a wide array of skills: technical (clinical), intellectual, and interpersonal-political. It is difficult to think of another subdiscipline within professional psychology that is positioned in such a challenging context. That psychologists worldwide are shouldering this burden is a testimony for the growing maturity of our field.

Second, in order for clinical psychologists in medical settings to be able to shoulder this burden, they need to think in an interdisciplinary manner. No longer is the divide between culture, mind, body, and brain feasible, and psychologists must reflect and converse in terms linking all the latter four domains.

Finally, and no least importantly, clinical psychologists in medical settings must be truly integrative (Shahar, 2013). This means that they need to shed all traditional allegiance to the One and True theoretical persuasion, and to implement interventions taken from a variety of worldviews: cognitive, behavioral, interpersonal, existential, psychodynamic, family system, and more. In that sense, it is my hope that this special issue on psychopathology in medical setting will contribute not only to the development of clinical-health psychology, but also to the integrative science and practice.

\section{Compliance with Ethical Standards}

Conflict of interest Author Golan Shahar declares that he has no conflict of interest.

Human and Animal Rights and Informed Consent No human or animal research studies were conducted by the authors for this article.

\section{References}

Ahmedani, B. K., Peterson, E. L., Hu, Y., Rossom, R. C., Lynch, F., Lu, C. Y., ... \& Waitzfelder, B. E. et al. (2017). Major physical health conditions and risk of suicide. American Journal of Preventive Medicine, 53, 308-315. https://doi.org/10.1016/j.amepr e.2017.04.001.

American Psychiatric Association. (2013). Diagnostic and statistical manual of mental disorders (5th ed.). Arlington, VA: American Psychiatric Publishing. 
Barber, S., \& Thornicroft, G. (2018). Reducing the mortality gap in people with severe mental disorders: The role of lifestyle psychosocial interventions. Frontiers in Psychiatry. https://doi. org/10.3389/fpsyt.2018.00463.

Bareket-Bojmel, L., Shahar, G., \& Margalit, M. (2020). COVID-19-related economic anxiety is as high as health anxiety: Findings from the USA, the UK, and Israel. International Journal of Cognitive Therapy. https://doi.org/10.1007/s41811-020-00078-3.

Beck, C. T., \& Gable, R. K. (2012). A mixed methods study of secondary traumatic stress in labor and delivery nurses. Journal of Obstetric, Gynecologic \& Neonatal Nursing, 41(6), 747-760.

Bolger, A., Collins, A., Michels, M., \& Pruitt, D. (2018). Characteristics and outcomes of children with conversion disorder admitted to a single inpatient rehabilitation unit. A retrospective study. $P M \& R, 10(9), 910-916$. https://doi.org/10.1016/j. pmrj.2018.03.004.

Bolton, C., Thilges, S., Lane, C., Lowe, J., \& Mumby, P. (2020). Posttraumatic stress disorder following acute delirium. Journal of Clinical Psychology in Medical Settings.

Bursch, B., Emerson, N. D., \& Sanders, M. J. (2020). Evaluation and management of Fictitious Disorder Imposed on Another. Journal of Clinical Psychology in Medical Settings.

Costello, L. H., Suh, C., Burnett, B., Kelsay, K., Bunik, M., \& Talmi, A. (2020). Addressing Adolescent Depression in Primary Care: Building Capacity through Psychologist and Pediatrician Partnership. Journal of Clinical Psychology in Medical Settings.

Dempsey, A. G., Chavis, L., Willis, T., Zuk, J., \& Cole, J. (2020). Addressing perinatal mental health risk within a fetal care center. Journal of Clinical Psychology in Medical Settings.

Doss, J. L., \& Plioplys, S. (2018). Pediatric psychogenic nonepileptic seizures: A concise review. Child and Adolescent Psychiatric Clinics of North America, 27(1), 53-61. https://doi.org/10.1016/j. chc.2017.08.007.

Fehr, K. K., Chambers, D. E., \& Ramasani, J. (2020). The impact of anxiety on behavioral sleep difficulties and treatment in young children: A Review of the Literature. Journal of Clinical Psychology in Medical Settings.

Joiner, T. E. (2005). Why people die by suicide. Harvard University Press.

Karoly, P. (2018). A goal-centered, self-regulatory model of motivation and its relevance for advancing the study of chronic pain. In P. Karoly \& G. Crombez (Eds.), Motivational perspectives on chronic pain: Theory, research, and practice (pp. 3-61). New York: Oxford University Press.

Karoly, P. (2020). How pain shapes depression and anxiety: A hybrid self-regulatory/predictive mind perspective. Journal of Clinical Psychology in Medical Settings.

Katon, W. J. (2011). Epidemiology and treatment of depression in patients with chronic medical illness. Dialogues in Clinical Neuroscience, 13(1), 7-23.

Kushnir, J., \& Sadeh, A. (2011). Sleep of preschool children with nighttime fears. Sleep Medicine, 12(9), 870-874.

Latremoliere, A., \& Woolf, C. J. (2009). Central sensitization: A generator of pain hypersensitivity by central neural plasticity. The Journal of Pain, 10(9), 895-926. https://doi.org/10.1016/j.jpain .2009.06.012.

Loughan, A., Lanoye, A., Aslanzadeh, F. J., Villanueva, A. A. L., Boutte, R., Husain, M., \& Braun, S. (2020). Fear of cancer recurrence and death anxiety: Unaddressed concerns for adult neuro-oncology patients. Journal of Clinical Psychology in Medical Settings.

Moon-Grady, A. J., Baschat, A., Cass, D., Choolani, M., Copel, J. A., Crombleholme, T. M., ... \& Norton, M. E. (2017). Fetal treatment 2017: the evolution of fetal therapy centers: A joint opinion from the international fetal medicine and surgical society (IFMSS) and the north-American fetal therapy network (NAFTNET). Fetal diagnosis and therapy, 42(4), 241-248.

Otis, J. D., Keane, T. M., Kerns, R. D., Monson, C., \& Scioli, E. (2009). The development of an integrated treatment for veterans with comorbid chronic pain and posttraumatic stress disorder. Pain Medicine, 10, 1300-1311.

Potthoff, L. M., Palma, I-A, F., Bujoreanu, I. S., \& Thompson, K. (2020). Admitted for an eating disorder: Challenges clinical psychologists face in working with patients and their families on a consultation-liaison service in a tertiary pediatric hospital. Journal of Clinical Psychology in Medical Settings.

Rodrigues, N., Shih, S., \& Cohen, L. L. (2019). Pica in pediatric sickle cell disease. Journal of Clinical Psychology in Medical Settings. https://doi.org/10.1007/s10880-019-09671-x.

Rogers, M. L., Joiner, T. E., \& Shahar, G. (2020). Suicidality in chronic illness: An overview of cognitive-affective and interpersonal factors. Journal of Clinical Psychology in Medical Settings

Shahar, G. (2013). Where we should be: An introduction to the special issue on psychotherapy integration in behavioral medicine. Journal of Psychotherapy Integration, 23, 1-4.

Shahar, G., Lassri, D., \& Luyten, P. (2014). Depression in chronic illness: A behavioral medicine approach. In D. Mostofsky (Ed.), Handbook of behavioral medicine (Chapter 1 (pp. 3-22). New York: Wiley.

Sim, L., Weber, C. H., Harrison, T., \& Peterson, C. (2020). Central sensitization in chronic pain and eating disorders: A potential shared pathogenesis. Journal of Clinical Psychology in Medical Settings.

Weisfeld, C. C., \& Dunleavy, K. (2020). Strategies for managing chronic pain, chronic PTSD, and comorbidities: Reflections on a case study documented over ten years. Journal of Clinical Psychology in Medical Settings. https://doi.org/10.1007/s10880-02009741-5.

Weiss, K. E., Steinman, K. J., Kodish, I., Sim, L., Yurs, S., Steggall, C., $\&$ Fobian, A. D. (2020). Functional neurological symptom disorder in children and adolescents within medical settings. Journal of Clinical Psychology in Medical Settings.

Woolf, C. J. (2011). Central sensitization: Implications for the diagnosis and treatment of pain. Pain, 52, S2-S15. https://doi. org/10.1016/j.pain.2010.09.030.

Yalom, I. D. (1980). Existential psychotherapy. New York: Guilford.

Zuckerbrot, R. A., Cheung, A. H., Jensen, P. S., Stein, R. E., \& Laraque, D. (2007). Guidelines for Adolescent Depression in Primary Care (GLAD-PC): I. Identification, assessment, and initial management. Pediatrics, 120(5), e1299-e1312. https://doi.org/10.1542/ peds.2007-1144.

Publisher's Note Springer Nature remains neutral with regard to jurisdictional claims in published maps and institutional affiliations. 